Acta Crystallographica Section D

Biological

Crystallography

ISSN 0907-4449

\section{Zbigniew Dauter}

Synchrotron Radiation Research Section, National Cancer Institute, NSLS, Brookhaven National Laboratory, Building 725A-X9, Upton, NY 11973, USA

Correspondence e-mail: dauter@bnl.gov

\title{
One-and-a-half wavelength approach
}

In many cases single-wavelength anomalous diffraction (SAD) phasing leads to a successful structure solution, but it is impossible to predict beforehand if single-wavelength data with a certain amount of anomalous signal resulting will be sufficient. It is therefore safer to continue collecting data at different wavelengths according to a MAD protocol, but to simultaneously attempt to phase the first data set by the SAD method. If this is successful, then further data collection can be abandoned. This '1.5-wavelength' approach may save a substantial amount of time and effort and diminishes the effects of crystal radiation damage. The principles of SAD phasing are illustrated using vector diagrams in the Argand plane.

\section{Introduction}

Currently, the most common way of solving novel macromolecular crystal structures is the multiwavelength anomalous diffraction (MAD) method (Smith \& Hendrickson, 2001). Instead of collecting diffraction data from the native crystal and a number of derivatives, as in the multiple isomorphous replacement (MIR) approach, the MAD technique is based on data sets, usually three or four, recorded at various wavelengths from only one kind of crystal containing suitable anomalous scatterers. Whereas in MIR the protein phases are estimated from the additional scattering of the heavy atoms present in the derivative structures, in MAD the protein phases are calculated from the wavelength-dependent quantitative differences in the anomalous scattering contribution of certain atoms contained in the crystal. There are two basic approaches to MAD phasing. The first, classic, method (Karle, 1980; Hendrickson, 1991) is based on the algebraic solution of the system of equations. The alternative method (Ramakrishnan \& Biou, 1997) applies the MIR phasing techniques, treating MAD as a special case of MIR.

Since the introduction of the MAD method (Karle, 1980; Phillips \& Hodgson, 1980; Hendrickson, 1991), the recommended procedures have evolved considerably. The first MAD structures have been solved with diffraction data collected at second-generation synchrotrons at ambient temperatures. Under these conditions, crystal diffraction decay has been a very significant factor. The strict procedures leading to good resulting phases involved collecting successively narrow batches of rotation images at several wavelengths in the inverse-beam mode. This ensured that the same reflections and their Friedel mates were measured under as similar conditions as possible at all wavelengths. The introduction of cryogenic cooling has changed these requirements, to a large extent alleviating the radiation-damage effects. It
Received 28 February 2002 Accepted 12 September 2002
(C) 2002 International Union of Crystallography Printed in Denmark - all rights reserved 
became more effective in terms of time and effort to collect a full data set at one wavelength before setting the monochromator to the next wavelength. The introduction of extremely bright third-generation synchrotron beamlines changed the situation again, since at APS, ESRF or SPring8 even frozen crystals undergo significant radiation damage within a few minutes of irradiation.

In parallel to the technological advances of cryocrystallography and synchrotron beamlines and, in addition, in the production of selenomethionine protein variants by genetic engineering, methodological progress in the phasing procedures has led to faster, more automatic and reliable algorithms and programs. Macromolecular crystallography has now evolved to such an extent that structural genomics projects, aiming at rapidly solving a large number of new structures in a short time, are actively and successfully pursued in many laboratories.

The necessity of rapid turnover of new crystal structures has led to efforts directed towards speeding up X-ray data collection and phasing procedures. One of the possibilities is to limit the number of data sets necessary to solve a novel crystal structure. It has been shown that only one-wavelength data suffice to solve the structure if the anomalous diffraction signal of the scatterers introduced to the crystal or inherently present within the macromolecule is measured accurately. Early examples include the use of the anomalous signal of sulfur for the solution of the structure of crambin (Hendrickson \& Teeter, 1981). Wang's simulations (Wang, 1985) suggested that an anomalous signal as small as $0.6 \%$ of the total scattering may be sufficient for successful phasing.

In general, there is no guarantee that a single-wavelength diffraction (SAD) approach will lead to the structure solution, but data collected at the first wavelength should be interpreted and the preliminary phasing performed as rapidly as possible. In favourable cases, collection of data at the second wavelength can be interrupted before completion. This would lead to the 'one-and-a-half wavelength' (1.5- $\lambda$ ) approach, which can be treated as SAD with an additional insurance policy.

\section{Examples of the 1.5- $\lambda$ approach}

An approach similar to $1.5-\lambda$ has been implicitly proposed by Rice et al. (2000), who reinterpreted several SeMet MAD structure solutions and concluded that the solutions could have been achieved by SAD using only one, peak-wavelength, data set. The authors suggested that in the MAD work the first-wavelength data with maximum anomalous signal should be collected particularly accurately, with high redundancy, before moving to the other wavelength.

A number of examples among the data collected at the X9B beamline at the National Synchrotron Light Source (Brookhaven National Laboratory, Upton, NY, USA) can be classified as the 1.5- $\lambda$ approach. Some of them will be briefly described here. In all examples, the diffraction images were interpreted and data processed with $H K L 2000$ (Otwinowski \& Minor, 1997).

\subsection{Glucuronyltransferase}

This structure has been solved from crystals of the SeMet variant (Pedersen et al., 2000). It contains two molecules of 261 amino acids, each containing three ordered Se atoms per asymmetric unit of the $P 2_{1}$ crystal. In the text of the publication, the authors stated that the structure has been solved by the SAD technique, whereas in fact the data have been collected at the next wavelength as well. However, the rapidly processed $1.6 \AA$ resolution data from the first, peak, wavelength displayed a very clear anomalous signal and the SAD structure solution with SHELXD (Sheldrick, 1998), MLPHARE (Otwinowski, 1993) and DM (Cowtan \& Zhang, 1999) up to the initial electron-density map display took less than half an hour, when the collection of data at the second wavelength was not yet halfway through. The course of this procedure is summarized in Table 1 and a fragment of the experimental map is shown in Fig. 1(a).

The interpretation of the first diffraction images was performed against the first exposures, so that the image integration proceeded parallel to the exposure of further images. The integration of the first-wavelength data set was finished very shortly after the end of all exposures. Although the structure analysis of glucuronyltransferase was not performed within the framework of high-throughput projects, it illustrates very clearly the possibility of such rapid structure determinations.

\subsection{Thioesterase}

The human acyl-protein thioesterase crystallizes with two molecules of $28 \mathrm{kDa}$ each in the asymmetric unit (Devedjiev $e t$ al., 2000). The crystal was soaked for a short time in cryosolution containing $1 \mathrm{M} \mathrm{NaBr}$ and the first diffraction data set was collected at the $\mathrm{Br}$ wavelength to a resolution of $1.8 \AA$. The anomalous signal was very clear and several bromide sites were quickly identified by $\operatorname{SnB}$ (Weeks \& Miller, 1999). Collection of data at the next wavelength was therefore abandoned. Six initially identified $\mathrm{Br}$ sites were expanded in three iterations of SHARP (de La Fortelle \& Bricogne, 1997) phasing to 22 sites; after density modification by $D M$, the figure of merit increased from 0.40 to 0.85 and the resulting map (Fig. 1b) showed clearly most of the protein chain. 347 out of the total 464 amino acids of the protein model were built automatically by wARP (Perrakis et al., 1999).

\subsection{PSCP}

The structure of Pseudomonas serine-carboxyl proteinase (PSCP) was previously solved from the anomalous signal of the cryosoaked bromides (Dauter et al., 2001). The crystals of PSCP contain one molecule with 372 amino acids in the asymmetric unit. In this case, only the $1.8 \AA$ resolution peakwavelength data were used for phasing, but the threewavelength MAD data were collected for the purpose of comparison of different phasing approaches. Nine $\mathrm{Br}$ sites identified by SHELXD were input to SHARP (FOM 0.21) and the resulting protein phases were improved by $D M$ (FOM 0.74), leading to a very clear map (Fig. 1c). The warpNtrace 
procedure automatically built 363 out of 372 residues with most of the correct side chains.

An analogous procedure where the full three-wavelength MAD data were used led to a SHARP FOM of 0.43 and $D M$ FOM of 0.63 , the latter value being lower than that obtained from the SAD phasing. However, the $D M$ procedure in the three-wavelength MAD phasing converged after four cycles, whereas in the SAD case it ran for seven cycles. Nevertheless, the MAD map was slightly superior to the SAD, although the SAD data collection and phasing required more than three times less time for the successful solution of the structure.

\section{Background of SAD}

Anomalous scattering is caused by the resonant effects of electrons whose excitation energy is close to the energy of the incident X-rays. It can be described by the additional corrections, real $\left(f^{\prime}\right)$ and imaginary $\left(f^{\prime \prime}\right)$, to the atomic scattering factor,

$$
f_{j}=f_{j}^{0}(\theta)+f_{j}^{\prime}(\lambda)+i f_{j}^{\prime \prime}(\lambda)
$$

where the normal scattering, $f_{j}^{0}(\theta)$, depends on the diffraction angle $\theta$ but not on the wavelength, and the anomalous corrections, real $f_{j}^{\prime}(\lambda)$ and imaginary $i f_{j}^{\prime \prime}(\lambda)$, depend on wavelength but not on the diffraction angle. If the crystal contains $A$ atoms diffracting anomalously and $N$ normal atoms

\section{Table 1}

Structure solution of glucuronidase.

\begin{tabular}{lc}
\hline Step & Time (min) \\
\hline SCALEPACK (3 times) & $6: 00$ \\
SHELXD & $2: 00$ \\
MLPHARE (FOM 0.19) & $1: 50$ \\
DM (10 cycles) (FOM 0.82) & $5: 45$ \\
FFT & $0: 30$ \\
QUANTA & $0: 30$ \\
Dead time & $\sim 8: 00$ \\
Total & $\sim 25: 00$ \\
\hline
\end{tabular}

then, neglecting the displacement parameters, the total structure factor for reflection $F_{T}(h)$ will be

$$
\begin{aligned}
F_{T}(h)= & \sum_{i}^{N} f_{i}^{0}(\theta) \exp \left[2 \pi i\left(h \cdot r_{i}\right)\right] \\
& +\sum_{j}^{A}\left[F_{j}^{0}(\theta)+f_{j}^{\prime}(\lambda)+i f_{j}^{\prime \prime}(\lambda)\right] \exp \left[2 \pi i\left(h \cdot r_{i}\right)\right] .
\end{aligned}
$$

This is illustrated in Fig. 2(a), where contributions from individual atoms of the structure are represented as vectors in the Argand plane. As seen in Fig. 2(b), the Friedel-related reflection, $F(-h)$, has all contributing vectors directed at the negated phase, except the imaginary contributions of the anomalously scattering atoms, which point in a direction $90^{\circ}$ more positive than that of the normal scattering of these atoms. That is strictly true if all anomalous scatterers are of the same kind, since then

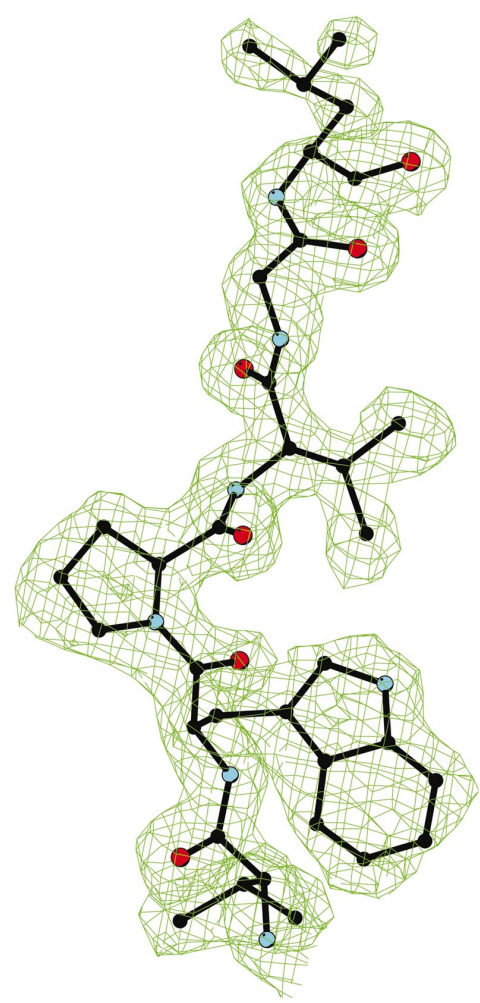

(a)

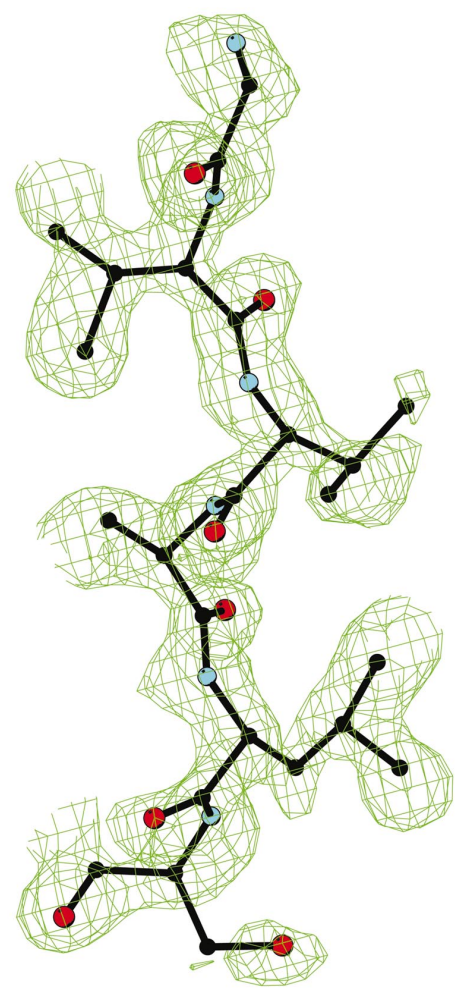

(b)

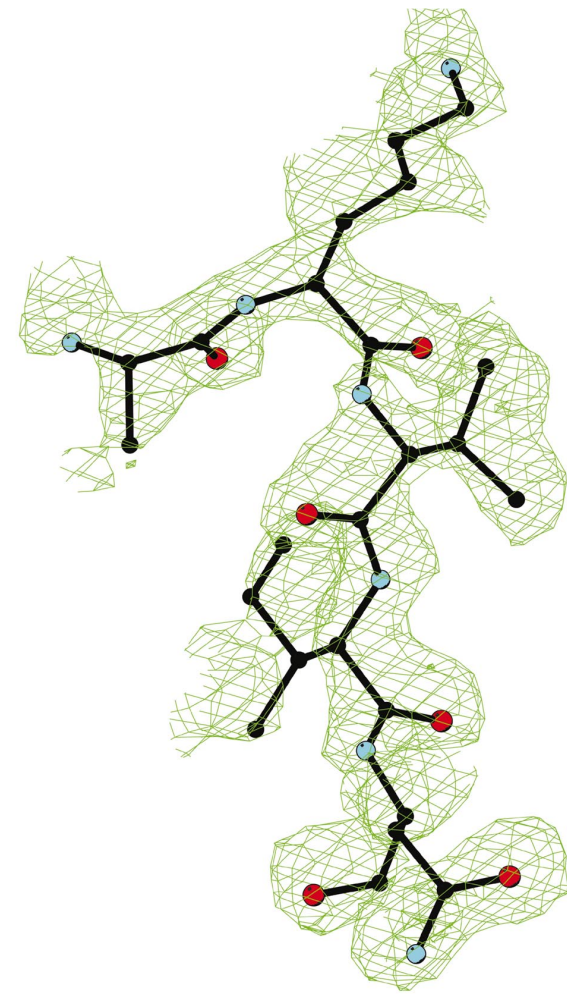

(c)

Figure 1

Regions of experimentally phased electron-density maps after solvent flattening, contoured at 1.5o. (a) Glucuronyltransferase, $(b)$ thioesterase, $(c)$ PSCP. 


$$
\begin{aligned}
F_{T}= & \sum_{i}^{N} f_{i}^{0}(\theta) \exp \left[2 \pi i\left(h \cdot r_{i}\right)\right] \\
& +\sum_{j}^{A}\left[f^{0}\left(1+f^{\prime} / f^{0}+i f^{\prime \prime} / f^{0}\right)\right] \exp \left[2 \pi i\left(h \cdot r_{i}\right)\right] \\
= & F_{N}+F_{A}+F_{A}^{\prime}+i F^{\prime \prime} .
\end{aligned}
$$

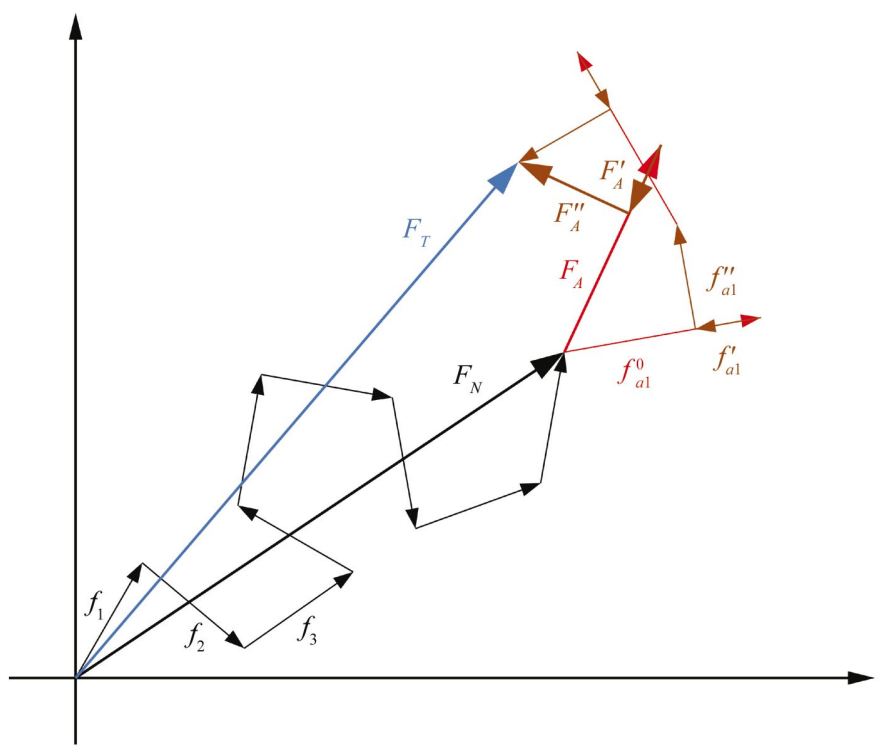

(a)

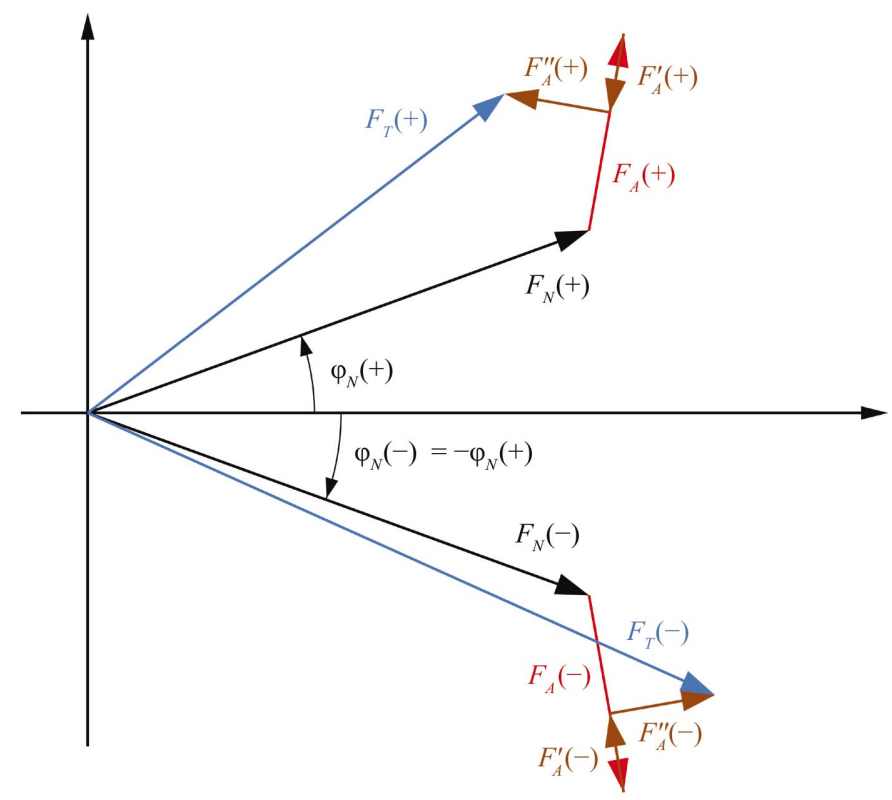

(b)
Otherwise, the vector corresponding to the total anomalous contribution, $F_{A}^{\prime \prime}$, is not perpendicular to that of the normal contribution, $F_{A}+F_{A}^{\prime}$. As a result, the total scattering vectors for the two Friedel mates $F(h)$ and $F(-h)$ differ in length and in phase if the crystal contains anomalous scatterers. It is customary to represent the vectors corresponding to the negative Friedel mate $F(-h)$ as reflected to the other side of the diagram, i.e. to draw its complex conjugate $* F(-h)$, since it visualizes more easily the phase relations between the two Friedel-related reflections (Fig. 2c).

The difference between amplitudes of two Friedel mates depends on the mutual disposition of the phases of the total scattering vector $F_{T}$ and those of the normal scattering of the anomalous scatterers $F_{A}$. Fig. 3 shows two extreme possibilities, where these two vectors are either parallel or perpendicular. In the former case, no Bijvoet difference is observed since both mates, $F_{T}^{+}$and $F_{T}^{-}$, have equal length. This is always true for centrosymmetric reflections, since all normal scattering vectors are purely real, mutually parallel or antiparallel (Fig. 4). For those reflections, the real $F_{A}^{\prime}$ contribution (usually negative) diminishes or increases the measured reflection amplitude if $F_{T}$ and $F_{A}$ have equal (both 0 or $180^{\circ}$ ) or opposite (one 0 and another $180^{\circ}$ ) phases, respectively. The significant imaginary $F_{A}^{\prime \prime}$ contribution always increases the measured centrosymmetric amplitudes, irrespective of the phase difference.

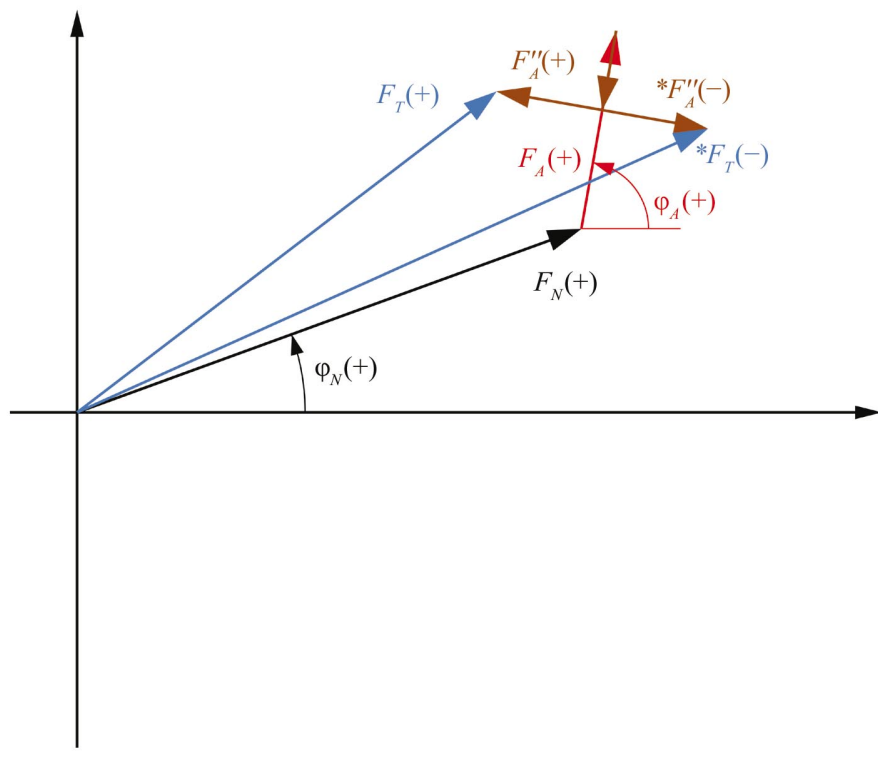

(c)

\section{Figure 2}

The structure factor of a reflection with its anomalous scattering contribution. In this and all subsequent figures, the contribution of normally scattering atoms is represented by black vectors, the normal scattering of anomalous scatterers by red vectors, the anomalous scattering (real and imaginary contributions) by brown vectors and the total structure factor by blue vectors. (a) The contributions of $N$ normally scattering atoms add up and can be represented by the vector $F_{N}=\sum_{i}^{N} f_{i}^{0} \exp 2 \pi i\left(h \cdot r_{i}\right)$. The $A$ anomalous scatterers contribute individually to the total normal diffraction $F_{A}=\sum_{j}^{A} f_{i}^{\prime} \exp \left[2 \pi i\left(h \cdot r_{j}\right)\right]$, the real part of the anomalous correction $F_{A}^{\prime}=\sum_{j}^{A} f_{j}^{\prime} \exp \left[2 \pi i\left(h \cdot r_{j}\right)\right]$ and the imaginary part of the anomalous correction $F_{A}^{\prime \prime}=\sum_{j}^{A} f_{j}^{\prime \prime} \exp \left[2 \pi i\left(h \cdot r_{j}\right)\right]$. If all anomalous scatterers are of the same kind, vector $F_{A}^{\prime \prime}$ preceeds $F_{A}$ by $90^{\circ}$. (b) The vector diagram for the Friedel pair, $F(h)$ and $F(-h)$. In relation to $F(h)$, all vectors representing the real contributions to the structure factor have negated phases, whereas the imaginary component $F_{h}^{\prime \prime}$ has its phase shifted by $90^{\circ}$ forward from $F_{A}$. (c) It is customary to reflect the vectors of the negative Friedel mate, $F(-h)$, across the horizontal axis and represent it as a complex conjugate, $F(-h)$, which more clearly illustrates the relations between vectors and phases of the Friedel mates. 


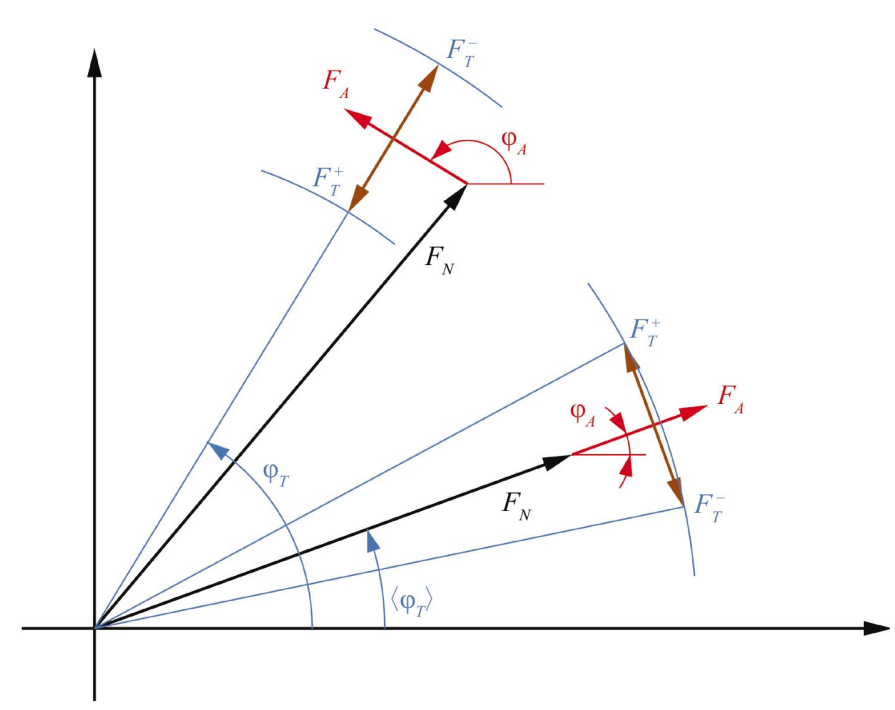

\section{Figure 3}

The Bijvoet difference between the amplitudes of Friedel mates depends on the difference between the phase of the normal scattering vector of the anomalous atoms, $\varphi_{A}$, and the phase of the average total structure factor, $\left\langle\varphi_{T}\right\rangle$. If these two phases differ by $90^{\circ}$, the Bijvoet difference is the largest possible; if they differ by 0 or $180^{\circ}$, no Bijvoet difference is observed.
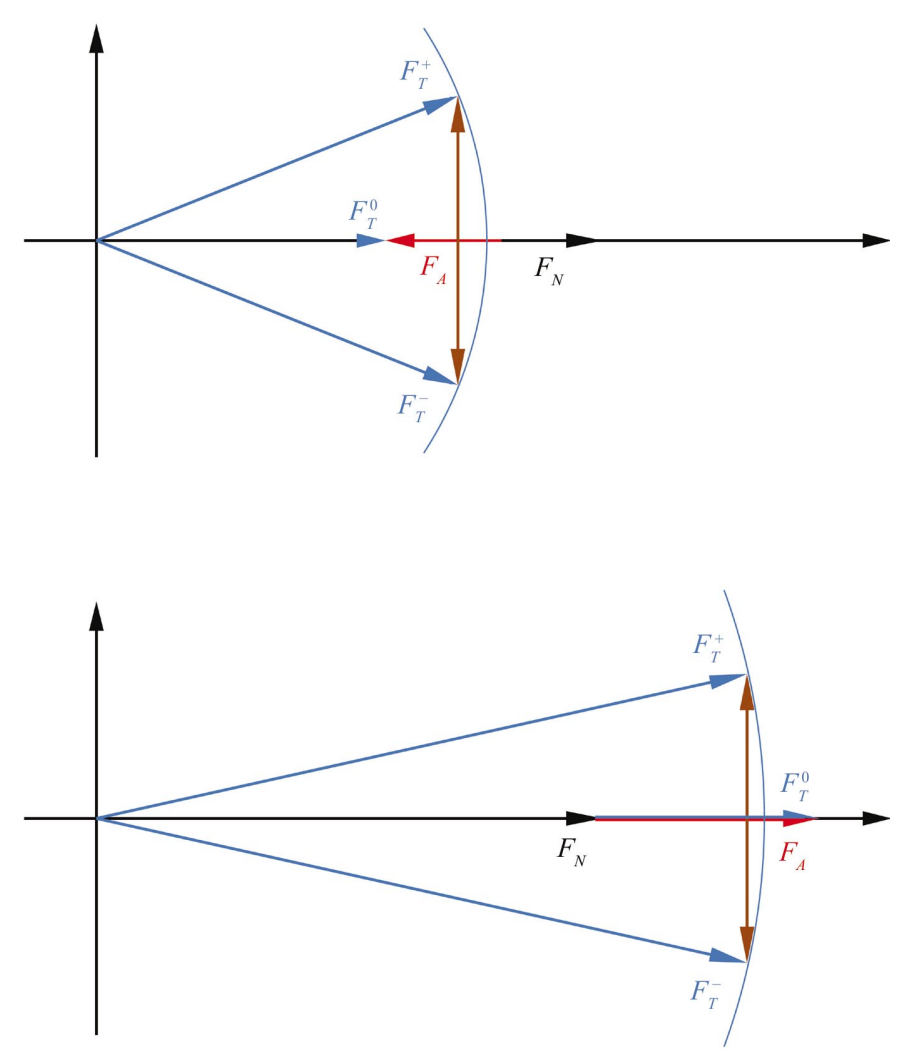

\section{Figure 4}

For a centrosymmetric reflection, both Friedel mate amplitudes are equal, since all normal scattering contributions are purely real. If vectors $F_{N}$ and $F_{A}$ are parallel (bottom diagram), the measured amplitude, $F_{T}^{+}=F_{T}^{-}$, tends to be similar to the amplitude $F_{T}^{0}$ measured at the wavelength at which the anomalous effect is negligible. If $F_{N}$ and $F_{A}$ vectors are antiparallel (top diagram), the measured total anomalous amplitude tends to be larger than that without the anomalous effect.
If the scattering vectors of the normal and anomalous atoms are perpendicular, $\varphi_{T}-\varphi_{A}= \pm 90^{\circ}$, the magnitude of the Bijvoet difference, $\left|F_{T}^{+}-F_{T}^{-}\right|$, is the largest possible. Theoretically, the Bijvoet differences should depend sinusoidally on the difference between the two phases (Hendrickson, 1979),

$$
\Delta F^{ \pm}=F^{+}-F^{-}=2 F_{A}^{\prime \prime} \sin \left(\varphi_{T}-\varphi_{A}\right)
$$

Fig. 5 shows this dependence for experimental diffraction data and phases calculated from the refined atomic model. The sinusoidal relationship is apparent in general, although both the measurement and model errors are significant.

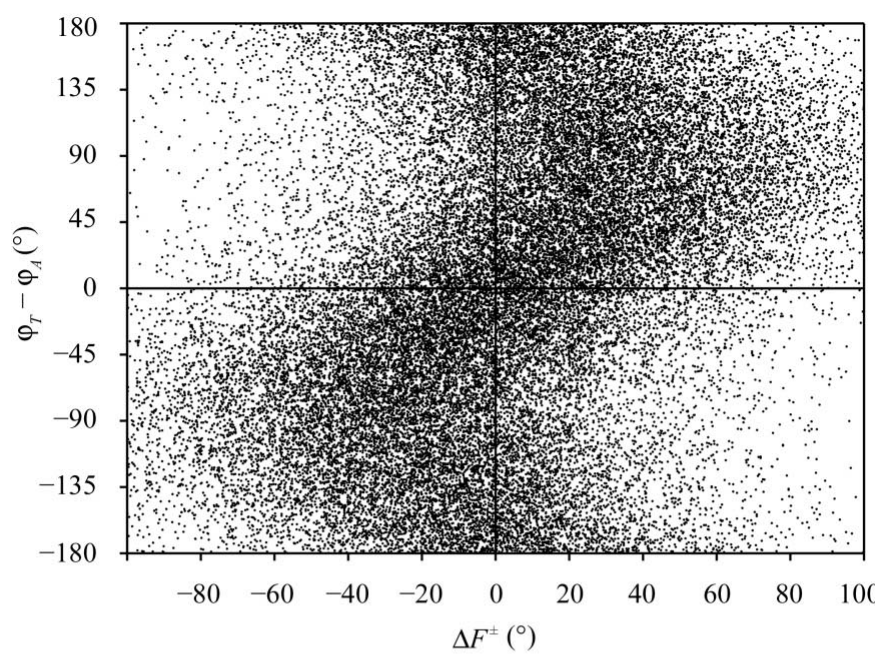

(a)

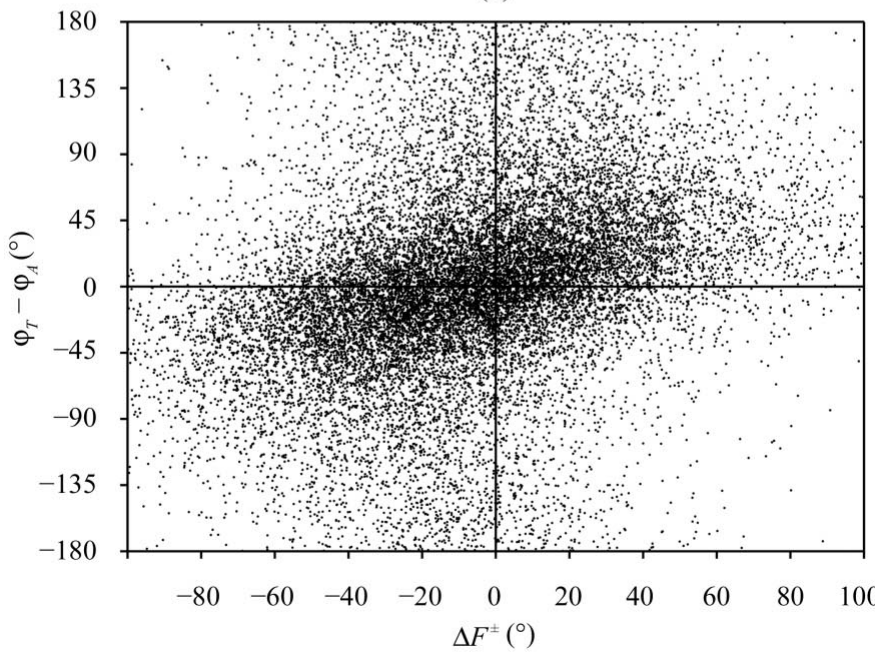

(b)

\section{Figure 5}

The relationship between the Bijvoet differences $\Delta F^{ \pm}$and the difference of two phases, that of the total scattering vector $\varphi_{T}$ and of the anomalous scatterers vector $\varphi_{A}$. The theoretically expected sinusoidal dependence, $\Delta F^{ \pm} \simeq \sin \left(\varphi_{T}-\varphi_{A}\right)$, is clearly reproduced for glucose isomerase $(a)$ with almost uniform phase distribution, whereas for ferredoxin $(b)$ the total protein phase tends to be closer to the anomalous phase. Glucose isomerase has one anomalous $\mathrm{Mn}$ atom in 388 residues and ferredoxin has eight anomalously scattering Fe atoms in 55 residues; therefore, the partial structure probability has a much larger effect in the case of ferredoxin than in glucose isomerase. 
To identify the positions of anomalous scatterers within the crystal, it is, in principle, necessary to extract their diffraction vectors $F_{A}$. This is not possible from one-wavelength data, where only two measurements, $F_{T}^{+}$and $F_{T}^{-}$, are available for each reflection. It becomes possible when additional data are collected at different wavelengths with different $F_{A}^{\prime}$ and/or $F_{A}^{\prime \prime}$ anomalous contributions. In a MAD experiment, several data sets collected with wavelengths around the absorption edge of the anomalous scatterer provide different magnitudes of $f^{\prime}$ and $f^{\prime \prime}$ (Fig. 6) and the relations between various contributing vectors and their phases can be solved analytically, as proposed by Karle (1980).

However, if only SAD data are available, it is possible to locate the anomalous scatterers using the largest Bijvoet differences instead of $F_{A}$ values. For reflections with the largest anomalous differences where the $\varphi_{T}-\varphi_{A}$ phase difference is close to $\pm 90^{\circ}$ (one of the cases in Fig. 3), the measured Bijvoet difference is proportional to $F_{A}$,

$$
\Delta F^{ \pm} \simeq 2 F_{A}^{\prime \prime}=2 F_{A}\left(f^{\prime \prime} / f^{0}\right),
$$

so that the large Bijvoet differences can be used in direct methods or Patterson searches to find positions of the anomalous scatterers. The use of Bijvoet differences to locate anomalous scatterers was first proposed by Rossmann (1961).

When positions of the anomalous scatterers are known, it is possible to calculate their contribution to the total diffraction. However, even in the ideal case of error-free measurements, this does not provide a unique solution to the phase problem, since in general there are two possible arrangements satisfying the vector relations. They lead to two possible total protein phase values $\varphi_{T}$ symmetrically placed around the $\varphi_{A}-90^{\circ}$, direction (Fig. 7a), also illustrated in Fig. 5. The possible values of $\varphi_{T}$ are (Ramachandran \& Raman, 1956)

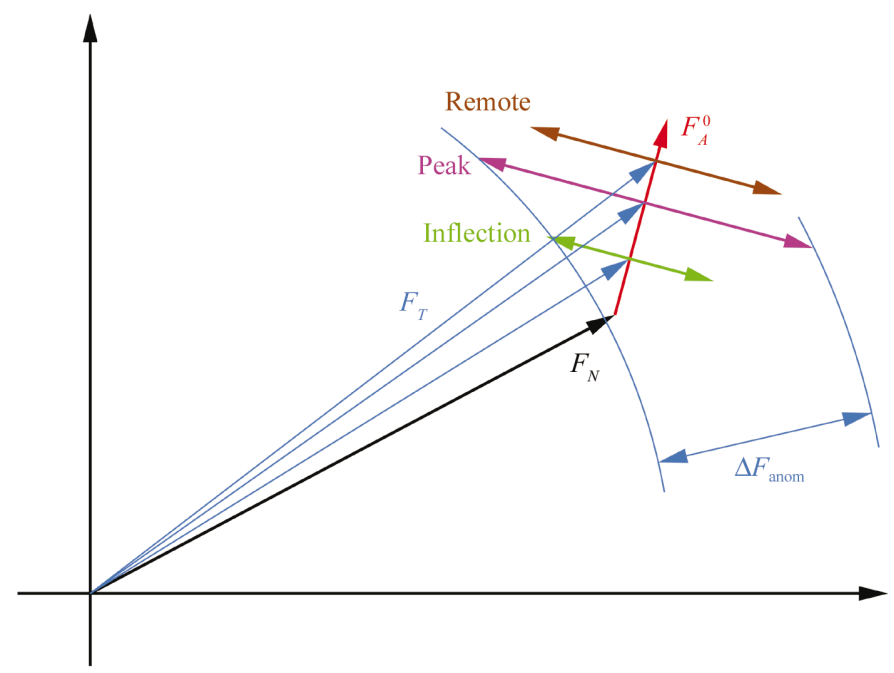

Figure 6

Anomalous-scattering contributions in the three MAD data sets. Data collected at the wavelength corresponding to the inflection point of the fluorescence spectrum have the maximum (most negative) $f^{\prime}$ and smallest $f^{\prime \prime}$ contributions. The remote-wavelength data have the smallest $f^{\prime}$ and medium $f^{\prime \prime}$ and the fluorescence-peak data have an intermediate $f^{\prime}$ but a maximum $f^{\prime \prime}$ value.

$$
\varphi_{T}=90^{\circ}+\varphi_{A} \pm \theta \text {, }
$$

where

$$
\theta=\cos ^{-1}\left(\Delta F^{ \pm} / 2 F_{A}^{\prime \prime}\right)
$$

Only for the largest Bijvoet differences does this SAD ambiguity degenerate to a single solution with $\varphi_{T}-\varphi_{A}= \pm 90^{\circ}$.

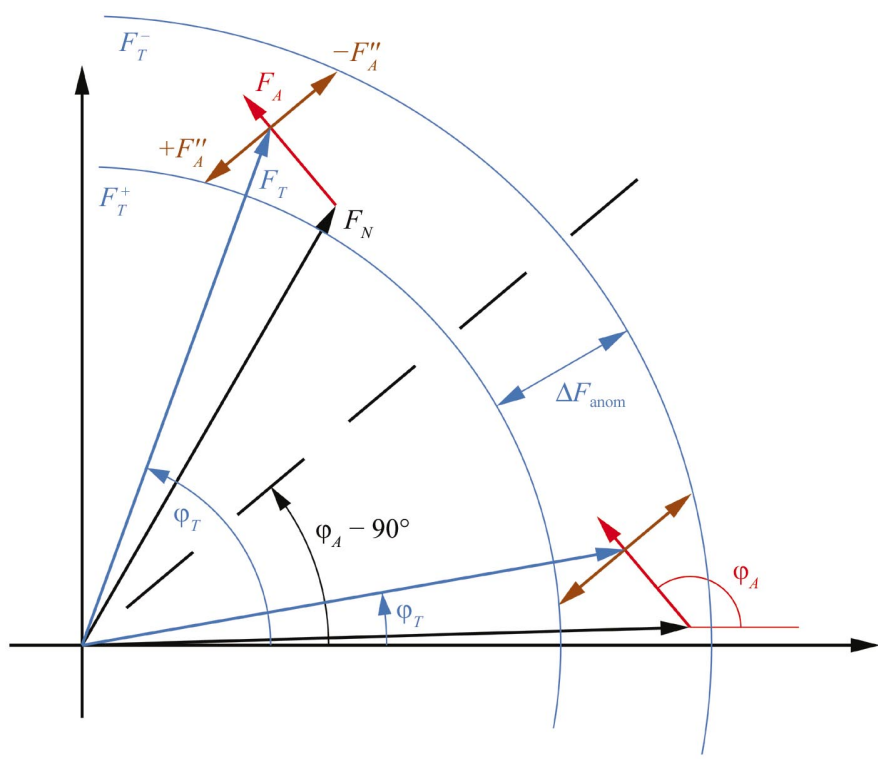

(a)

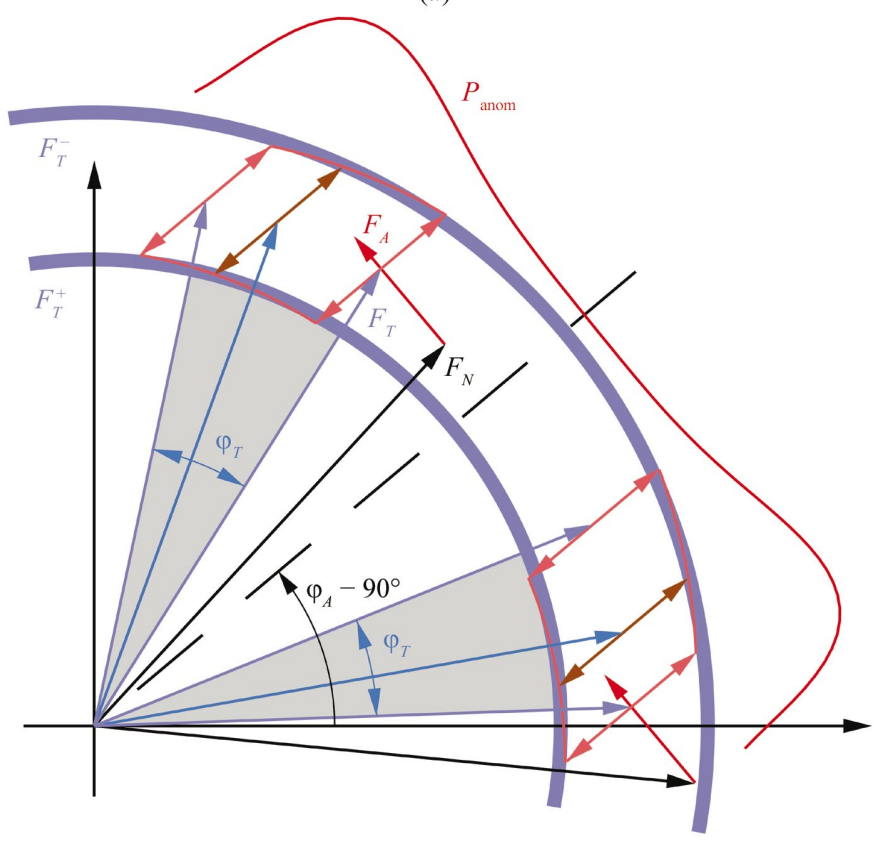

(b)

Figure 7

The SAD phase ambiguity. If the positions of anomalous scatterers are known, their scattering contribution can be calculated. (a) With the accurately measured amplitudes of two Friedel mates, $F_{T}^{+}$and $F_{T}^{-}$, there are two possible solutions for the total protein phase $\varphi_{T}$ placed symmetrically around $\varphi_{A}-90^{\circ}$, the direction of the anomalous vector $F_{A}^{\prime \prime}$. (b) If the measurement errors of the Friedel amplitudes are taken into account, the protein phase can be described by a predictable probability distribution. 
The reflection intensities measured in a diffraction experiment inevitably contain errors. The anomalous scattering signal is usually small, at the level of a few percent of the total crystal diffraction. The anomalous diffraction data should therefore be collected very carefully with proper estimation of the errors (standard uncertainties) associated with the measured intensities. If measurement errors are taken into account, the modified SAD vector diagram, Fig. 7(b), shows

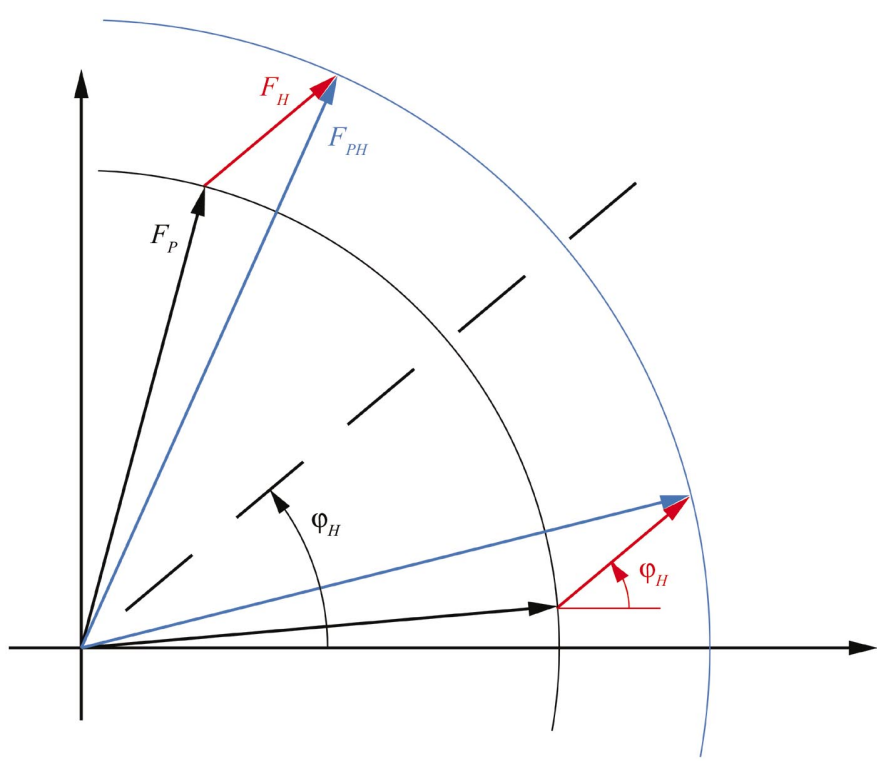

(a)

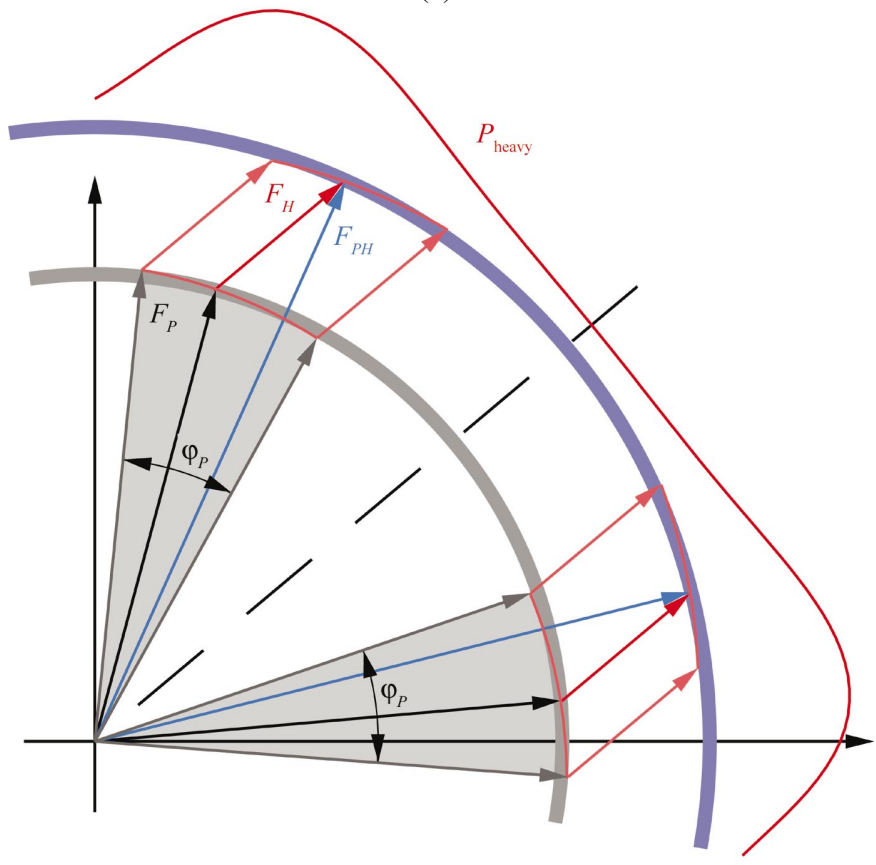

(b)

Figure 8

Like the SAD case, there is also an ambiguity in single isomorphous replacement (SIR). (a) With the measured amplitudes of the native protein $\left(F_{P}\right)$ and heavy-atom derivative $\left(F_{P H}\right)$ amplitudes and the calculated heavy-atom contribution $\left(F_{H}\right)$, there are in general two possible solutions for the protein phase $\varphi_{P}$ symmetrically placed around the heavy-atom phase $\varphi_{H}$. $(b)$ The error treatment leads to the probability distribution of the protein phase. that instead of two sharp solutions there are two regions where the total protein phase $\varphi_{T}$ may lie. The probability that the protein phase has a particular value, given the known Bijvoet difference $\Delta F^{ \pm}$, calculated anomalous scatterers phase $\varphi_{A}$ and imaginary contribution $F_{A}^{\prime \prime}$, is (Hendrickson \& Teeter, 1981)

$$
P_{\text {anom }}\left(\varphi_{T}\right)=N \exp \left\{-\left[\Delta F^{ \pm}+2 F_{A}^{\prime \prime} \sin \left(\varphi_{T}-\varphi_{A}\right)\right]^{2} / 2 E^{2}\right\},
$$

where $E$ is the standard error and $N$ the normalizing factor. This can also be expressed using the Hendrickson-Lattman (Hendrickson \& Lattman, 1970) coefficients,

$$
\begin{aligned}
P_{\text {anom }}\left(\varphi_{T}\right)= & N \exp \left(A \cos \varphi_{T}+B \sin \varphi_{T}\right. \\
& \left.+C \cos 2 \varphi_{T}+D \sin 2 \varphi_{T}\right)
\end{aligned}
$$

or, since this function is symmetric around $\varphi_{A}-90^{\circ}$,

$$
\begin{aligned}
P_{\text {anom }}\left(\varphi_{T}\right)= & N \exp \left[S \cos \left(\varphi_{T}-\varphi_{A}+90^{\circ}\right)\right. \\
& \left.+T \cos \left(\varphi_{T}-\varphi_{A}+90^{\circ}\right)\right] .
\end{aligned}
$$

The above analysis of the phasing information based on the single-wavelength anomalous signal is analogous to the single isomorphous replacement (SIR) case, where only the native and a single derivative data are available. Again, the heavy atoms can be located using the isomorphous differences $F_{P H}-F_{P}$ and SIR also leads to an ambiguity, with two possible protein phases symmetrically placed around the heavy-atom phase $\varphi_{H}$ (Fig. $8 a$ ). The SIR error treatment, originally derived by Blow \& Crick (1959), leads to a similar equation for the protein phase probability, $P_{\text {heavy }}\left(\varphi_{P}\right)$, which can be expressed similarly in terms of Hendrickson-Lattman coefficients (Fig. 8b).

The SIR ambiguity can be broken by the use of the second derivative, when only one solution for each derivative should coincide, indicating the proper protein phase (Fig. 9a). In the case of anomalous data, this can be achieved by the use of data collected at different wavelengths, where the anomalous scattering contributions are different (Fig. 9b). This is the concept of the multiwavelength (MAD) approach. However, the changes in the real, $f^{\prime}$, and imaginary, $f^{\prime \prime}$, contributions play different roles in MAD phasing. $f^{\prime \prime}$ (in relation to $\Delta F^{ \pm}$) indicates the positions of the two alternative phase solutions, which differ for the second wavelength only if the $f^{\prime}$ value is different; otherwise, the possible solutions coincide for both wavelengths, even if the $f^{\prime \prime}$ values are different. Therefore, only the differences in $f^{\prime}$ indicate which alternative is correct.

The analogy between SAD and SIR is not complete. Comparison of Figs. $7(a)$ and $8(a)$ shows that whereas all vectors in the SIR case, $F_{P}, F_{H}$ and $F_{P H}$, are of equal length in both alternative solutions, in the SAD case the $F_{T}$ and $F_{A}$ vectors have the same length, but the scattering of the normal atoms $F_{N}$ is different. This is more clearly seen in Fig. 10, where in one solution the $F_{N}$ vector is required to be much longer than in the other and significantly longer than the total scattering vector $F_{T}$; the latter situation is less probable. The anomalous scatterers can be treated as the known partial structure. The theory of protein phase probability with known partial structure has been worked out by Sim $(1959,1964)$, 


$$
P_{\text {part }}\left(\varphi_{T}\right)=N \exp \left\{2\left[\left(F_{T} F_{A}\right) / F_{U}^{2}\right] \cos \left(\varphi_{T}-\varphi_{A}\right)\right],
$$

where $F_{U}$ is the scattering of the unknown part of the structure and $N$ is the normalizing factor.

The probability resulting from the known partial structure depends on the cosine function of the phase difference $\varphi_{T}-\varphi_{A}$ and as a result the combined probability is different for the

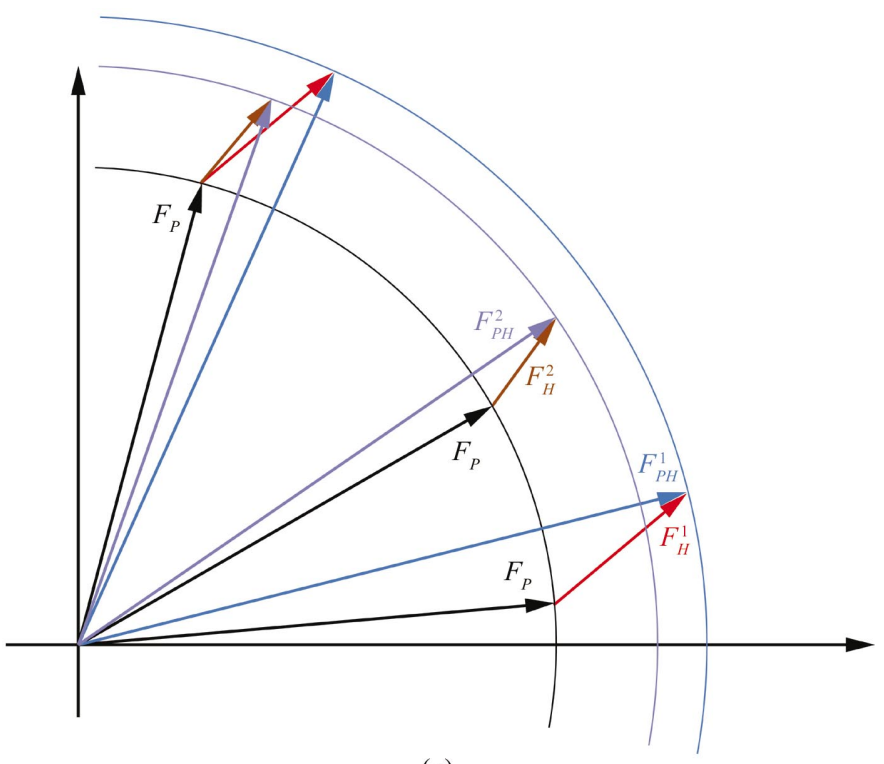

(a)

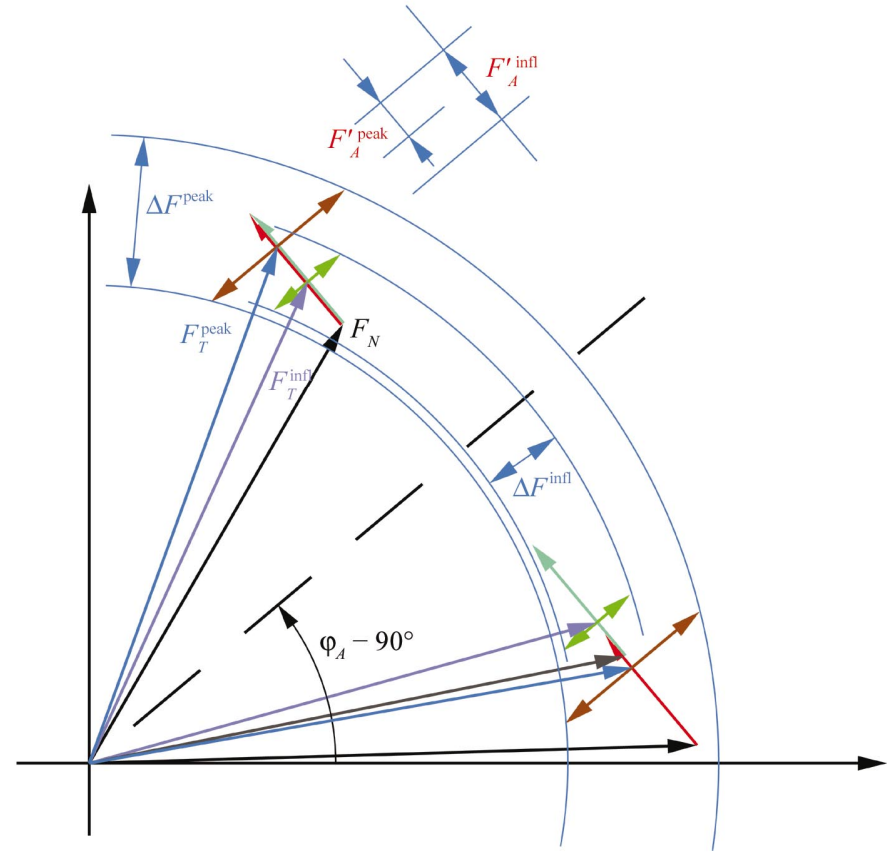

(b)

\section{Figure 9}

The second derivative in the isomorphous replacement $(a)$ or the secondwavelength data in MAD $(b)$ indicates which alternative solution is correct. In MAD it is, however, necessary that the $f^{\prime}$ contribution has to be different at the two wavelengths; otherwise, the selection cannot be achieved, even if $f^{\prime \prime}$ values are different. Therefore, in the MAD approach the $f^{\prime \prime}$ values indicate the two possible values of the protein phase and the dispersive differences in $f^{\prime}$ provide an indication which of the two alternatives is correct. two alternative solutions for $\varphi_{T}$ (Fig. 11). Ramachandran \& Raman (1956) first postulated that the total phase closer to the anomalous phase should be chosen for map calculation, which agrees with the Sim probability indication based on the partial structure.

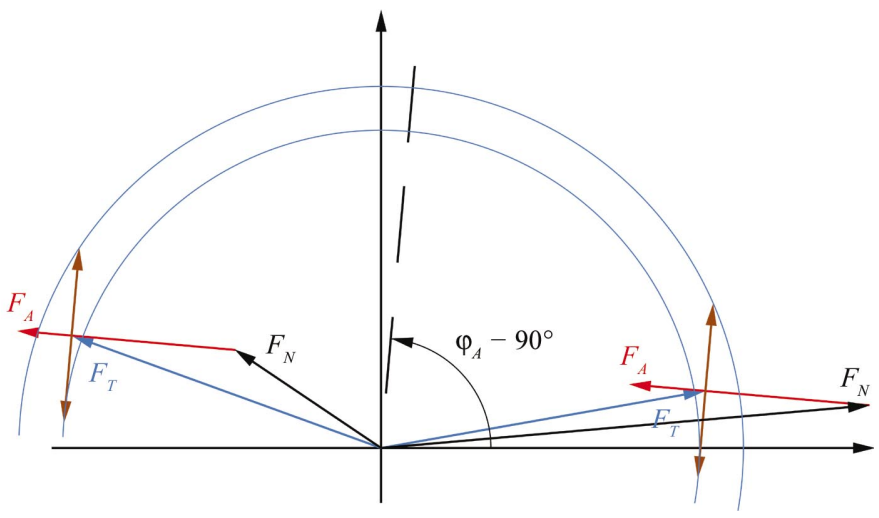

\section{Figure 10}

The two alternative SAD phase diagrams are not fully symmetric, since they involve different contributions of the normal scatterers. When the anomalous and total phases are similar (as on the left side of the graph) the normal scatterers amplitude is smaller than when the difference between these two phases is close to $180^{\circ}$. This difference can be quantitatively described by the effect of the known partial structure (of the anomalous scatterers) on the probability distribution of the total protein phase.

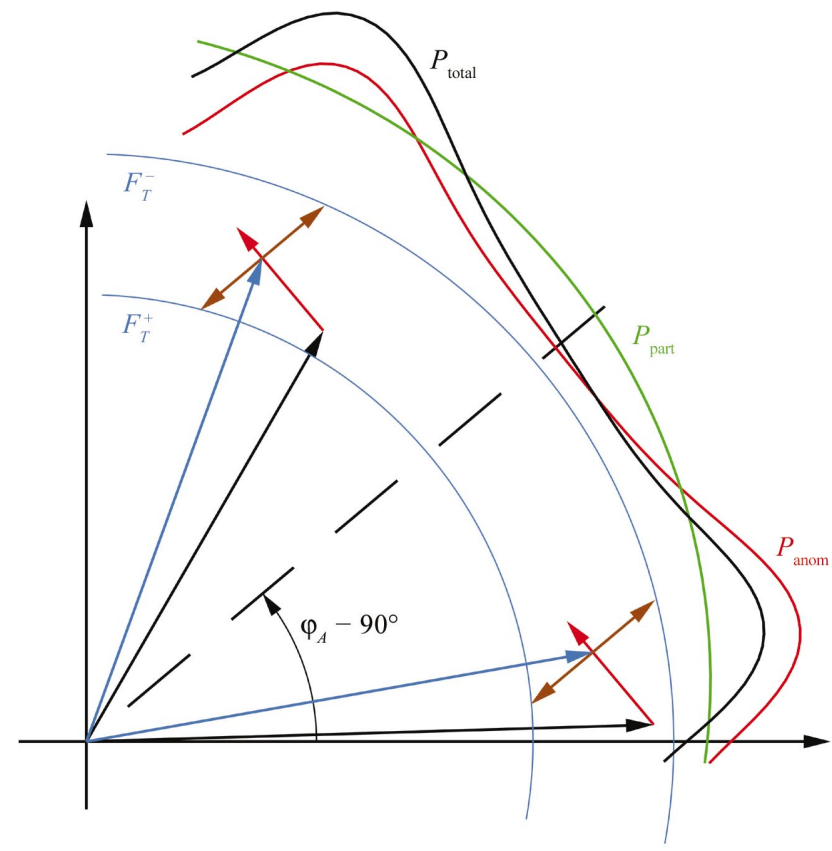

Figure 11

If the probabilities resulting from the anomalous scattering and from the partial structure are combined, the total probability distribution indicates that the SAD solution where the total phase is closer to the anomalous scatterers phase is more likely to be correct. This effect is clearly visible for ferredoxin (Fig. $5 b$ ), where the partial structure of the two $4 \mathrm{Fe}-4 \mathrm{~S}$ clusters is substantial, in contrast to glucose isomerase (Fig. 5a), where the single $\mathrm{Mn}$ atom constitutes only a small part of the whole structure. 


\section{SAD phasing}

The first practical use of the above relations was realised in the solution of the structure of crambin (Hendrickson \& Teeter, 1981) by the application of partial structure-resolved anomalous scattering. The positions of six S atoms were located from the anomalous difference Patterson map. The reflections with largest $\Delta F^{ \pm}$and unimodal probability distributions gave phases $\varphi_{A} \pm 90^{\circ}$ and other reflections according to the partial structure probability discrimination. Figures of merit were used for weighting in the map calculations. The correct enantiomer was chosen on the basis of the reasonableness of the Fourier map.

In his classic work, Wang (1985) proposed the iterative single-wavelength anomalous scattering (ISAS) approach. This takes advantage of the additive property of the Fourier transform. If the Fourier synthesis is calculated with structure factors corresponding to the sum of two alternative SAD vectors, the map should contain the proper crystal structure features superimposed on more or less uniform noise, since such vectors represent the sum of the correct structure factors leading to the protein map and of the wrong factors producing only the featureless noise (Fig. 12). In the first approximation, it should be possible to identify in such a map regions corresponding to the protein in contrast to flatter solvent regions. The protein features can then be enhanced by iterative noise filtering, a procedure which evolved into the well known solvent flattening. Moreover, this procedure effectively discriminates between two enantiomeric solutions.

The maximum-likelihood approach based on the twodimensional integration of phase and amplitude probabilities and rigorous error treatment is realised in SHARP (de La

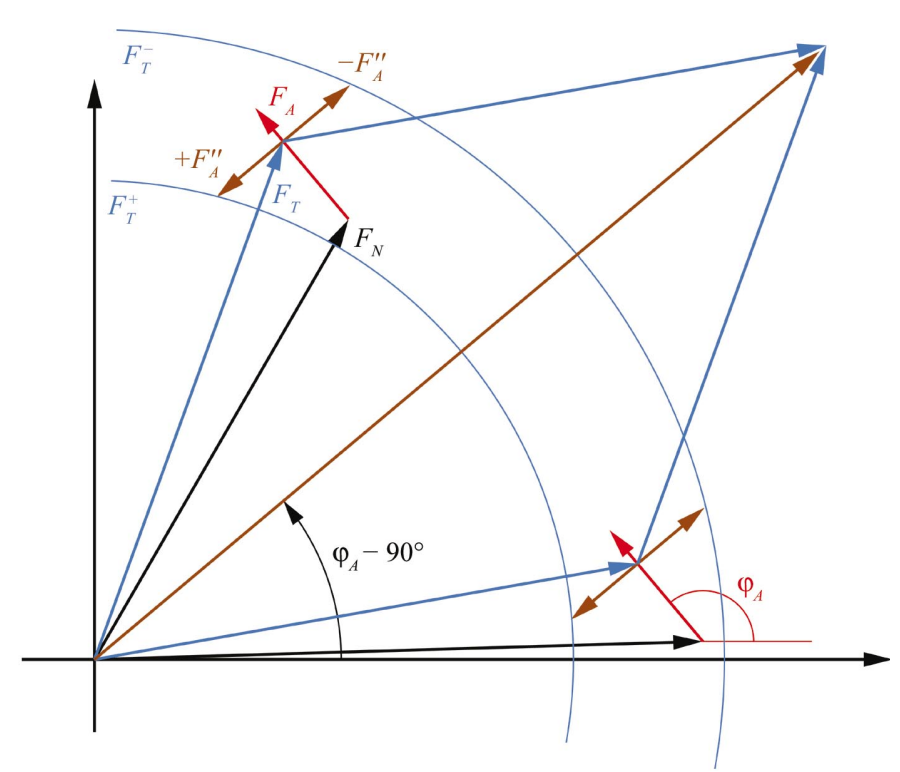

Figure 12

Illustration of the Wang's ISAS method. The Fourier map based on the structure factor corresponding to the vector sum of the two alternative SAD solutions should show the correct protein image resulting from the correct component vectors overlapped on the noise density resulting from the wrong components. The repetitive Fourier filtering of the noise in the solvent regions leads to a clear protein image.
Fortelle \& Bricogne, 1997). This allows the bias-free refinement of anomalous scatterers and other parameters, and simultaneous estimation of protein phases and their weights. Several other programs, e.g. MLPHARE (Otwinowski, 1993), SOLVE (Terwilliger \& Berendzen, 1999) and CNS (Brünger $e t$ al., 1998), treat SAD phasing as a special case of MIR or MAD and have been successfully used for this purpose.

A different approach is based on the application of direct methods, relying on the statistical relations between phases and Bijvoet differences of selected reflections (e.g. Hauptman, 1997; Langs et al., 1999; Liu et al., 1999). One such method is implemented in the program OASIS (Hao et al., 2000).

\section{Conclusions}

Numerous examples obtained in last couple of years show that SAD phasing is more powerful than had been anticipated. Various anomalous scatterers have been used (Hendrickson \& Ogata, 1997; Dauter et al., 2002), ranging from phosphorus and sulfur to selenium, halides, heavy metals and lanthanides. Clearly, the success of this approach critically depends on the accuracy of the anomalous signal contained in the diffraction data. The data-resolution limit seems to play a less important role, although it is obviously best to have accurate data at high resolution. As in the other approaches, the success of SAD cannot be guaranteed a priori; it therefore seems reasonable to continue collecting diffraction data at further wavelengths with MAD phasing in mind, but in parallel to attempt to solve the structure against the first data set using the SAD approach. With the currently available programs, one can perform this rapidly and, if successful, it may allow one to abandon the collection of further data. If the solution cannot be achieved by SAD, one can continue to perform the full multiwavelength experiment. Such a 1.5-wavelength approach may lead to less radiation damage to the crystal and to a substantial saving in time and effort, which is important for the currently pursued high-throughput structural projects.

Lars Pedersen is thanked for his kind permission to use glucuronyltransferase as an example of $1.5-\lambda$ phasing.

\section{References}

Blow, D. M. \& Crick, F. H. C. (1959). Acta Cryst. 12, 794-802.

Brünger, A. T., Adams, P. D., Clore, G. M., DeLano, W. L., Gros, P., Grosse-Kunstleve, R. W., Jiang, J. S., Kuszewski, J., Nilges, M., Pannu, N. S., Read, R. J., Rice, L. M., Simonson, T. \& Warren, G. L. (1998). Acta Cryst. D54, 905-921.

Cowtan, K. D. \& Zhang, K. Y. J. (1999). Prog. Biophys. Mol. Biol. 72, 245-270.

Dauter, Z., Dauter, M. \& Dodson, E. J. (2002). Acta Cryst. D58, 494 506.

Dauter, Z., Li, M. \& Wlodawer, A. (2001). Acta Cryst. D57, 239-249.

Devedjiev, Y., Dauter, Z., Kuznetsov, S. R., Jones, T. L. Z. \& Derewenda, Z. S. (2000). Structure, 8, 1137-1146.

Hao, Q., Gu, Y. X., Zheng, C. D. \& Fan, H. F. (2000). J. Appl. Cryst. 33, 980-981.

Hauptman, H. A. (1997). Curr. Opin. Struct. Biol. 7, 672-680.

Hendrickson, W. A. (1979). Acta Cryst. A35, 245-247.

Hendrickson, W. A. (1991). Science, 254, 51-58. 
Hendrickson, W. A. \& Lattman, E. E. (1970). Acta Cryst. B26, 136143.

Hendrickson, W. A. \& Ogata, C. M. (1997). Methods Enzymol. 276, 494-523.

Hendrickson, W. A. \& Teeter, M. M. (1981). Nature (London), 290, 107-113.

Karle, J. (1980). Int. J. Quant. Chem. Symp. 7, 357-367.

La Fortelle, E. de \& Bricogne, G. (1997). Methods Enzymol. 276, 472494.

Langs, D. A., Blessing, R. H. \& Guo, D. Y. (1999). Acta Cryst. A55, 755-760.

Liu, Y. D., Harvey, I., Gu, Y. X., Zheng, C. D., He, Y., Fan, H., Hasnain, S. S. \& Hao, Q. (1999). Acta Cryst. D55, 1620-1622.

Otwinowski, Z. (1993). Proceedings of the CCP4 Study Weekend. Isomorphous Replacement and Anomalous Scattering, edited by W. Wolf, P. R. Evans \& A. G. W. Leslie, pp. 80-86. Warrington: Daresbury Laboratory.

Otwinowski, Z. \& Minor, W. (1997). Methods Enzymol. 276, 307-326.

Pedersen, L. C., Tsuchida, K., Kitagawa, H., Sugahara, K., Darden, T. A. \& Negishi, M. (2000). J. Biol. Chem. 275, 34580-34585.
Perrakis, A., Morris, R. J. \& Lamzin, V. S. (1999). Nature Struct. Biol. 6, 458-463.

Phillips, J. C. \& Hodgson, K. O. (1980). Acta Cryst. A36, 856-864. Ramachandran, G. N. \& Raman, S. (1956). Curr. Sci. 25, 348-351.

Ramakrishnan, V. \& Biou, V. (1997). Methods Enzymol. 276, 538557.

Rice, L. M., Earnest, T. N. \& Brunger, A. T. (2000). Acta Cryst. D56, 1413-1420.

Rossmann, M. G. (1961). Acta Cryst. 14, 383-388.

Sheldrick, G. M. (1998). Direct Methods for Solving Macromolecular Structures, edited by S. Fortier, pp. 401-411. Dordrecht: Kluwer Academic Publishers.

Sim, G. A. (1959). Acta Cryst. 12, 813-815.

Sim, G. A. (1964). Acta Cryst. 17, 1072-1073.

Smith, J. L. \& Hendrickson, W. A. (2001). International Tables for Crystallography, Vol. F, edited by M. G. Rossmann \& E. Arnold, pp. 299-303. Dordrecht: Kluwer Academic Publishers.

Terwilliger, T. C. \& Berendzen, J. (1999). Acta Cryst. D55, 849-861. Wang, B. C. (1985). Methods Enzymol. 115, 90-112.

Weeks, C. M. \& Miller, M. (1999). J. Appl. Cryst. 32, 120-124. 